Boletim Científico do Instituto Agronômico do Estado de S. Paulo

Vol. 31

Campinas, novembro de 1972

N. ${ }^{\circ} 27$

\title{
ADUBAÇÃO DO TRIGO
}

\section{II - EXPERIENCIAS COM N, P, K E S EM LATOSSOLO VERMELHO ESCURO ORTO, NA REGIÃO SUL DO ESTADO DE SÃO PAULO $(1,2)$}

Carlos Eduardo de Oliveira Camargo, engenheiro-agiônomo $\left({ }^{3}\right)$, Seção de Arroz e Cereais de Inverno, Instituto Agronômico

\section{SINOPSE}

Em Latossolo Vermelho Escuro orto, de baixa fertilidade e até então não adubado, foram realizados dois ensaios de adubação $N, P, K$ e $S$ em cultura de trigo (Triticum aestivum L.), em áreas diferentes do município de Itapeva, SP, nos anos de 1969 e 1970. Empregou-se delineamento Central Composto.

Os dados obtidos mostraram grandes efeitos do nitrogênio, fósforo, potássio e enxofre.

Foram calculadas as quantidades de nitrogênio e de fósforo para a obtenção de maior lucro por área, para as relações de preços entre $\mathrm{N}$ e $\mathrm{P}-1,5: 1,1,33: 1$ e $1: 1$ - considerando-se fixa a dose de $30 \mathrm{~kg} / \mathrm{ha}$ de $\mathrm{K}_{2} 0$, e calculadas as quantidades de $K_{2} 0$ necessárias para a obtenção de maior lucro por área, para diferentes relações de preços do potássio, com um suprimento constante de $60 \mathrm{~kg} / \mathrm{ha}$ de $\mathrm{N}$ e $60 \mathrm{~kg} / \mathrm{ha}$ de $\mathrm{P}_{2} \mathbf{0}_{5}$.

\section{1 - INTRODUÇÃO}

O sul do Estado de São Paulo, tradicional região tritícola, vem diminuindo gradativamente sua área de cultivo, e um dos principais motivos é a baixa fertilidade dos solos, embora a região seja ecologicamente favorável à cultura, o mesmo acontecendo com a topografia, que permite a mecanização.

(1) Recebido para publicação em 15 de dezembro de 1971.

$\left({ }^{2}\right)$ o autor agradece aos engenheiros-agrônomos Derly Machado de Souza e Luiz Torres de Miranda, a orientação e o estímulo na realização do trabalho.

$\left({ }^{3}\right)$ Com bolsa de suplementação do CNPq. 
Segundo Gargantini (7), a cultura do trigo na referida região apresenta baixa produtividade, o que é atribuído à muito baixa porcentagem de saturação em bases, à excessiva pobreza em fósforo e à elevada acidez do solo.

Frattini (6) informa que em 1969 os municípios de Itapeva e Itaberá foram os de maior área plantada na região sul.

Considerando os problemas existentes é que se propôs executar o presente trabalho, onde são apresentados os resultados de duas experiências conduzidas no município de Itapeva, nos anos de 1969 e 1971, em áreas de Latossolo Vermelho Escuro orto, nas quais a adubação mineral $\mathrm{N}, \mathrm{P}, \mathrm{K}$ e S foi correlacionada com a produção de grãos.

\section{2 - MATERIAIS E METODOS}

O delineamento estatístico empregado foi o Central Composto $(\mathbf{l})$.

Os oito primeiros pontos correspondem a um fatorial $2^{3}$. Os sete seguintes são pontos axiais, incluindo um ponto no centro do delineamento.

Foram incluídos três tratamentos extras $(16,17$ e 18), os quais, com o tratamento 9, permitem avaliar o efeito do enxofre (S) .

Foram utilizadas duas repetições por local.

Como fonte de adubo nitrogenado foi utilizado o Nitrocálcio $(27 \% \mathrm{~N})$; como fonte de adubo fosforado foi utilizado o superfosfato triplo $\left(46 \%\right.$ de $\left.\mathrm{P}_{2} \mathrm{O}_{5}\right)$; como fonte de adubo potássico foi utilizado o cloreto de potássio $\left(60 \%\right.$ de $\left.\mathrm{K}_{2} \mathrm{O}\right)$; e como fonte de enxofre foi utilizado o gesso $(20 \% \mathrm{~S})$.

O modo de aplicação dos adubos obedeceu o seguinte critério: 4/5 do nitrogênio foram aplicados a $0,20 \mathrm{~m}$ de profundidade, correspondendo a uma aplicação na sola do arado; e o restante $(1 / 5)$ foi aplicado juntamente com o fósforo, potássio e enxofre, no sulco de plantio (3).

O cultivar teste foi o IRN-526-63, de origem mexicana. 
Os tratamentos foram os seguintes $(\mathrm{kg} / \mathrm{ha})$ :

Tratamento: $\quad \mathrm{N} \quad \mathrm{P}_{2} \mathrm{O}_{5} \quad \mathrm{~K}_{2} \mathrm{O} \quad \mathrm{S}$

\begin{tabular}{|c|c|c|c|}
\hline$\ldots \ldots$ & 30 & 30 & 15 \\
\hline $2 \ldots \ldots$ & 30 & 30 & 45 \\
\hline$\ldots \ldots \ldots$ & 30 & 90 & 15 \\
\hline$\ldots$ & 30 & 90 & 45 \\
\hline$\ldots \ldots \ldots$ & 90 & 30 & 15 \\
\hline$\ldots$ & 90 & 30 & 45 \\
\hline & 90 & 90 & 15 \\
\hline$\ldots \ldots \ldots$ & 90 & 90 & 45 \\
\hline$\ldots \ldots \ldots$ & 60 & 60 & 30 \\
\hline$\cdots$ & 120 & 60 & 30 \\
\hline$\ldots \ldots$ & 0 & 60 & 30 \\
\hline & 60 & 120 & 30 \\
\hline$\cdots$ & 60 & 0 & 30 \\
\hline$\ldots$ & 60 & 60 & 60 \\
\hline & 60 & 60 & 0 \\
\hline$\ldots \ldots \ldots$ & 0 & 0 & 0 \\
\hline$\ldots$ & 0 & 0 & 0 \\
\hline$\ldots \ldots$ & 60 & 60 & 30 \\
\hline
\end{tabular}

As parcelas foram constituídas de 10 linhas de 5 metros, espaçadas de $0,20 \mathrm{~m}$. A semeadura foi feita na base de 40 sementes úteis por metro de sulco, equivalendo a 2000 sementes úteis por parcela. Por ocasião da colheita foram colhidas as seis linhas centrais de cada parcela, deixando as demais como bordadura, portanto com uma área útil de $6 \mathrm{~m}^{2}$.

As duas experiências foram conduzidas em áreas diferentes, do município de Itapeva, S P. 
O primeiro ensaio foi semeado em 18 de abril e colhido em 17 de setembro de 1969, na Fazenda Barreirinho. O segundo foi semeado em 13 de abril e colhido em 25 de agosto de 1971, na Fazenda Primavera.

Foram retiradas amostras compostas dos solos dos locais estudados, cujos resultados analíticos $\left({ }^{4}\right)$ foram os seguintes:

\begin{tabular}{|c|c|c|}
\hline & F.B. & F.I \\
\hline $\mathrm{pH}$ int & 5,55 & 5,30 \\
\hline $\mathrm{C} \% \ldots$ & 1,40 & 1,8 \\
\hline $\mathrm{K}^{2+}\left({ }^{5}\right) \ldots \ldots$ & 0,15 & 0,10 \\
\hline $\mathrm{Ca}^{2+} \mathrm{Mg}^{2+}\left({ }^{5}\right)$ & 2,10 & 1,20 \\
\hline $\mathrm{PO}_{4}^{3-}\left({ }^{6}\right) \quad \ldots$ & 0,03 & 0,02 \\
\hline $\mathrm{Al}^{3+}\left({ }^{5}\right)$. & - & 1,00 \\
\hline
\end{tabular}

Foram calculadas as quantidades de nitrogênio e de fósforo necessárias para a obtenção do maior lucro por área, para as relações de preços entre $\mathrm{N}_{\text {e }} \mathrm{P}_{2} \mathrm{O}_{5}$ 1,5:1 e 1:1, considerando-se fixa a dose de $30 \mathrm{~kg} /$ ha de $\mathrm{K}_{2} \mathrm{O}$, e as quantidades de potássio necessárias para a obtenção do maior lucro por área, considerando-se fixas as doses de $60 \mathrm{~kg} / \mathrm{ha}$ de nitrogênio e $60 \mathrm{~kg} / \mathrm{ha}$ de $\mathrm{P}_{2} \mathrm{O}_{5}$.

\section{3 - RESULTADOS E DISCUSSÃO}

Nos dois ensaios foi satisfatória a germinação das sementes nas diferentes parcelas.

As produções de grãos por tratamento, nos anos de 1969 e 1971, acham-se no quadro 1 .

No quadro 2 encontram-se os resultados da análise estatística, na qual foram calculados os parâmetros da equação da experiência de 1969, os parâmetros da equação da experiência de 1971, bem como os parâmetros médios dos dois experimentos.

\footnotetext{
( ${ }^{4}$ Análise efetuada na Seção de Fertilidade do Solo, Instituto Agronômico.

${ }^{5}$ ) e.mg/100 g de solo. Teores trocáveis

( ${ }^{6}$ ) e.mg/100 g de solo. Teor solúvel em $\mathrm{H}_{2} \mathrm{SO}_{4} 0,05 \mathrm{~N}$
} 
Quadro 1. - Produções de grãos de trigo obtidas em duas experiências de adubação N, P, K e S realizadas em 1969 e 1971, em Latossolo Vermelho Escuro orto, no município de Itapeva, SP

\begin{tabular}{|c|c|c|c|c|c|c|}
\hline \multicolumn{4}{|c|}{ Tratamento } & \multicolumn{3}{|c|}{ Produção } \\
\hline $\mathbf{N}$ & $\mathrm{P}_{2} \mathbf{0}_{5}$ & $\mathrm{~K}_{2} \mathrm{O}$ & $\mathbf{S}$ & 1969 & 1971 & Média \\
\hline & & & & $k g / h a$ & $k g / h a$ & $k g / h a$ \\
\hline 30 & 30 & 15 & 40 & 1109 & 1430 & 1269 \\
\hline 30 & 30 & 45 & 40 & 1150 & 1240 & 1195 \\
\hline 30 & 90 & 15 & 40 & 1350 & 1880 & 1615 \\
\hline 30 & 90 & 45 & 40 & 1484 & 1740 & 1612 \\
\hline 90 & 30 & 15 & 40 & 575 & 2050 & 1313 \\
\hline 90 & 30 & 45 & 40 & 1392 & 1740 & 1566 \\
\hline 90 & 90 & 15 & 40 & 1325 & 1820 & 1573 \\
\hline 90 & 90 & 45 & 40 & 1563 & 2620 & 2092 \\
\hline 60 & 60 & 30 & 40 & 1250 & 2290 & 1770 \\
\hline 120 & 60 & 30 & 40 & 1434 & 2350 & 1892 \\
\hline 0 & 60 & 30 & 40 & 1075 & 1680 & 1378 \\
\hline 60 & 120 & 30 & 40 & 1525 & 2290 & 1908 \\
\hline 60 & 0 & 30 & 40 & 1138 & 1210 & 1174 \\
\hline 60 & 60 & 60 & 40 & 1450 & 2240 & 1845 \\
\hline 60 & 60 & 0 & 40 & 1417 & 1660 & 1538 \\
\hline 0 & 0 & 0 & 0 & 467 & 980 & 723 \\
\hline 0 & 0 & 0 & 40 & 563 & 1040 & 803 \\
\hline 60 & 60 & 30 & 0 & 1304 & 2080 & 1692 \\
\hline
\end{tabular}

A produção calculada é dada pela seguinte equação: $\mathrm{Y}=\mathrm{B}_{0}+\mathrm{B}_{1} \mathrm{x}_{1}+\mathrm{B}_{2} \mathrm{x}_{2}+\mathrm{B}_{3} \mathrm{x}_{3}+\mathrm{B}_{11} \mathrm{x}_{1}{ }^{2}+\mathrm{B}_{22} \mathrm{x}_{2}^{2}+\mathrm{B}_{33} \mathrm{x}_{3}^{2}$ $+\mathrm{B}_{12} \mathrm{x}_{1} \mathrm{x}_{2}+\mathrm{B}_{23} \mathrm{x}_{2} \mathrm{x}_{3}+\mathrm{B}_{13} \mathrm{x}_{1} \mathrm{x}_{3}$, onde $\mathrm{x}_{1}$ varia de $+2,+1,0,-1$, -2 , correspondendo respectivamente a $120,90,60,30,0 \mathrm{~kg}$ de $\mathrm{N}$ por hectare; $\mathrm{x}_{2}$ varia de $+2,+1,0,-1,-2$, correspondendo respectivamente a $120,90,60,30,0 \mathrm{~kg}$ de $\mathrm{P}_{2} \mathrm{O}_{5}$ por hectare; $\mathrm{x}_{3}$ varia de $+2,+1,0,-1,-2$, correspondendo respectivamente a 60,45 , $30,15,0 \mathrm{~kg}$ de $\mathrm{K}_{2} \mathrm{O}$ por hectare; $\mathrm{B}_{0}$ é a produção calculada para a dose central, ou seja, $\mathrm{x}_{1}=0, \mathrm{x}_{2}=0$ e $\mathrm{x}_{3}=0 ; \mathrm{B}_{1}$ é o efeito linear 


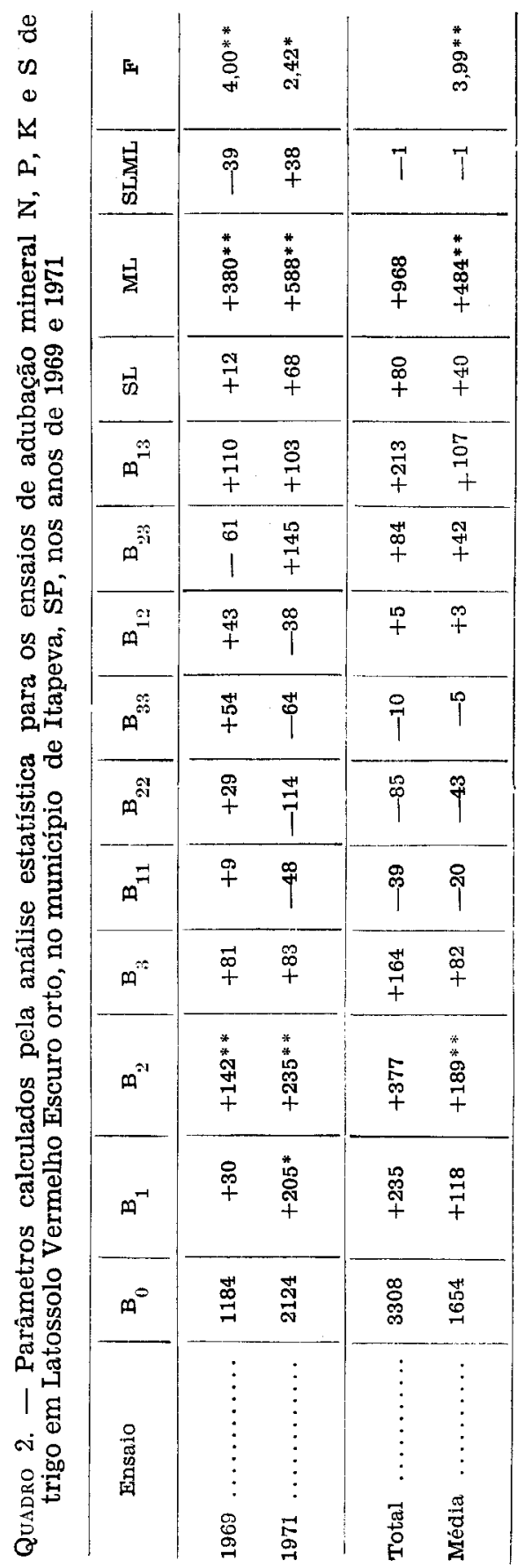


da nitrogênio; $\mathrm{B}_{2}$ é o efeito linear 'do $\mathrm{P}_{2} \mathrm{O}_{5} ; \mathrm{B}_{3}$ é o efeito linear do $\mathrm{K}_{2} \mathrm{O} ; \mathrm{B}_{12}$ é a interação $\mathrm{N}$ com $\mathrm{P}_{2} \mathrm{O}_{5} ; \mathrm{B}_{23}$ é a interação $\mathrm{P}_{2} \mathrm{O}_{5}$ com $\mathrm{K}_{2} \mathrm{O}, \mathrm{B}_{13}$ é a interação $\mathrm{N}$ com $\mathrm{K}_{2} \mathrm{O}$.

Se se considerar a equação média dos dois experimentos, verificar-se-á que o efeito linear do nitrogênio foi de +118 ; isto quer dizer que, para cada $30 \mathrm{~kg}$ de $\mathrm{N}$, há um acréscimo de 118 quilos na produção; o efeito linear do fósforo foi altamente significativo e igual a + 189; isto quer dizer que para cada $30 \mathrm{~kg}$ de $\mathrm{P}_{2} \mathrm{O}_{5}$ há um acréscimo de $189 \mathrm{~kg}$ na produção; o efeito linear de potássio foi de + 82; isto quer dizer que para cada $15 \mathrm{~kg}$ de $\mathrm{K}_{2} \mathrm{O}$ há um acréscimo de 82 quilos na produção.

Os efeitos quadráticos e interações na média dos dois experimentos foram respectivamente negativos e positivos, porém não significativos.

Considerando os tratamentos 9,16, 17 e 18, pode-se calcular o efeito da adubação mineral, que foi de $484 \mathrm{~kg} / \mathrm{ha}$, altamente significativo ao nível de $1 \%$; o efeito do enxofre foi positivo mas não estatisticamente significativo.

A análise conjunta dos dois experimentos revela um efeito de tratamentos significativo ao nível de $1 \%$, um efeito de anos também significativo ao nível de $1 \%$, porém interação tratamentos $\mathrm{x}$ anos não significativa .

Observando a figura 1 pode-se elaborar a relação seguinte, determinando as quantidades de nitrogênio e de fósforo necessárias para obtenção de maior lucro ou menor prejuízo por área, para as relações de preços entre $N$ e $P$ 1,5:1 e $1: 1$, permanecendo fixa a dose de $30 \mathrm{~kg}$ de $\mathrm{K}_{2} \mathrm{O}$ por hectare.

\begin{tabular}{ccccc}
\multicolumn{2}{c}{$\begin{array}{c}\text { Valores relativos por quilo } \\
(1 \mathrm{~kg} \text { de trigo }=1)\end{array}$} & \multicolumn{2}{c}{$\begin{array}{c}\text { Quantidade a ser } \\
\text { aplicada, em } \mathrm{kg} / \mathrm{ha}\end{array}$} \\
$\mathrm{N}$ & $\mathrm{P}_{2} \mathrm{O}_{5}$ & Trigo & $\mathrm{N}$ & $\mathrm{P}_{2} \mathrm{O}_{5}$ \\
5,25 & 3,50 & 1 & 40 & 86 \\
4,16 & 2,77 & 1 & 60 & 96 \\
3,00 & 2,00 & 1 & 84 & 104 \\
3,50 & 3,50 & 1 & 75 & 88 \\
2,77 & 2,77 & 1 & 88 & 98 \\
2,00 & 2,00 & 1 & 114 & 106 \\
0 & 0 & 1 & 144 & 129
\end{tabular}


A relação indica que: quando são necessários $5,25 \mathrm{~kg}$ de trigo para pagar $1 \mathrm{~kg}$ de $\mathrm{N}$ e $3,50 \mathrm{~kg}$ de trigo para pagar $1 \mathrm{~kg}$ de $\mathrm{P}_{2} \mathrm{O}_{5}$, a adubação que daria maior lucro por área é de $40 \mathrm{~kg}$ de $\mathrm{N}$ mais $86 \mathrm{~kg}$ de $\mathrm{P}_{2} \mathrm{O}_{5}$ por hectare; quando são necessários $4,16 \mathrm{~kg}$ de trigo para pagar $1 \mathrm{~kg}$ de $\mathrm{N}$ e 2,77 kg de trigo para pagar $1 \mathrm{~kg}$ de $\mathrm{P}_{2} \mathrm{O}_{5}$, a adubação que daria maior lucro por área é de $60 \mathrm{~kg}$ de $\mathrm{N}$ mais $96 \mathrm{~kg}$ de $\mathrm{P}_{2} \mathrm{O}_{5}$ por hectare; quando são necessários $3,0 \mathrm{~kg}$ de trigo para pagar $1 \mathrm{~kg}$ de $\mathrm{N}$ e $2,0 \mathrm{~kg}$ de trigo para pagar $1 \mathrm{~kg}$ de $\mathrm{P}_{2} \mathrm{O}_{5}$, a adubaçăo que daria maior lucro por área é de $84 \mathrm{~kg}$ de $\mathrm{N}$ mais $104 \mathrm{~kg}$ de $\mathrm{P}_{2} \mathrm{O}_{5}$ por hectare; quando são necessários $3,5 \mathrm{~kg}$ de trigo para pagar $1 \mathrm{~kg}$ de $\mathrm{N}$ e $3,5 \mathrm{~kg}$ de trigo para pagar $1 \mathrm{~kg}$ de $\mathrm{P}_{2} \mathrm{O}_{5}$, a adubação que daria maior lucro por área é de $75 \mathrm{~kg}$ de $\mathrm{N}$ mais $88 \mathrm{~kg}$ de $\mathrm{P}_{2} \mathrm{O}_{5}$ por hectare; quando são necessários $2,77 \mathrm{~kg}$ de trigo para pagar $1 \mathrm{~kg}$ de $\mathrm{N}$ e 2,77 kg de trigo para pagar $1 \mathrm{~kg}$ de $\mathrm{P}_{2} \mathrm{O}_{5}$, a adubação que daria maior lucro por área é de $88 \mathrm{~kg}$ de $\mathrm{N}$ mais 98 $\mathrm{kg}$ de $\mathrm{P}_{2} \mathrm{O}_{5}$ por hectare; quando são necessários $2 \mathrm{~kg}$ de trigo para pagar $1 \mathrm{~kg}$ de $\mathrm{N}$ e $2 \mathrm{~kg}$ de trigo para pagar $1 \mathrm{~kg}$ de $\mathrm{P}_{2} \mathrm{O}_{5}$, a adubação que daria maior lucro por área é de $114 \mathrm{~kg}$ de $\mathrm{N}$ mais $106 \mathrm{~kg}$ de $\mathrm{P}_{2} \mathrm{O}_{5}$ por hectare.

Para a obtenção da produção máxima possível, sem considerar os preços dos adubos, são necessários $144 \mathrm{~kg}$ de N mais $129 \mathrm{~kg}$ de $\mathrm{P}_{2} \mathrm{O}_{5}$ por hectare.

Os cálculos econômicos para determinar as quantidades de potássio a serem aplicadas para obtenção do maior lucro por área, para diferentes relações de preços do potássio para o trigo e na presença de $60 \mathrm{~kg}$ de $\mathrm{N}$ e $60 \mathrm{~kg}$ de $\mathrm{P}_{2} \mathrm{O}$ s por hectare, acham-se na relação dada a seguir.

Dessa relação pode-se inferir que: quando forem necessários $4 \mathrm{~kg}$ de trigo para comprar $1 \mathrm{~kg}$ de $\mathrm{K}_{2} \mathrm{O}$, aplicar-se-ão $63 \mathrm{~kg}$ de $\mathrm{K}_{2} \mathrm{O}$ por hectare; quando forem necessários $3 \mathrm{~kg}$ de trigo para comprar $1 \mathrm{~kg}$ de $\mathrm{K}_{2} \mathrm{O}$, aplicar-se-ão $85 \mathrm{~kg}$ de $\mathrm{K}_{2} \mathrm{O}$ por hectare; quando forem necessários $2 \mathrm{~kg}$ de trigo para comprar $1 \mathrm{~kg}$ de $\mathrm{K}_{2} \mathrm{O}$, apli- 


\section{CAMARGO}

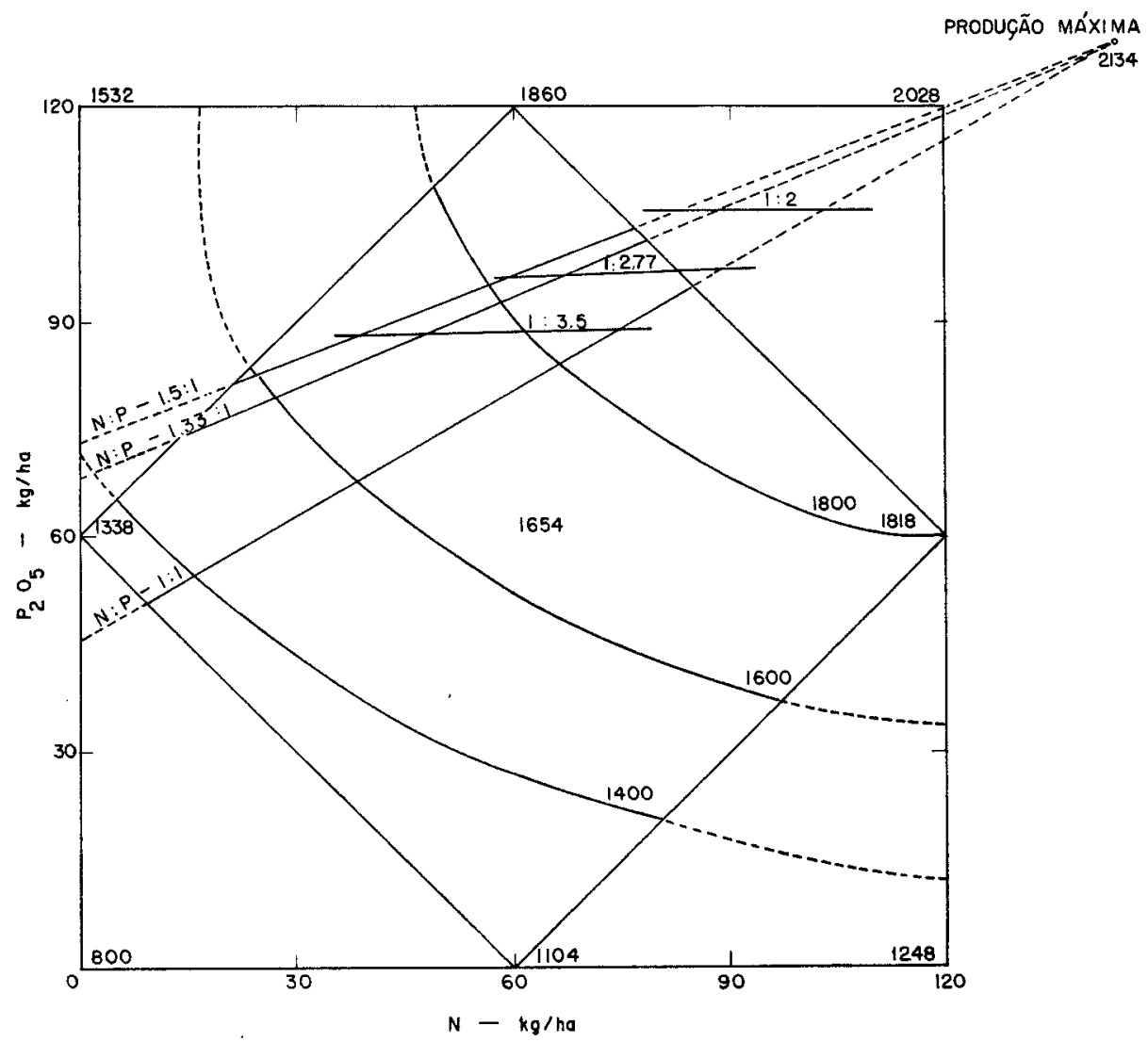

FIGURA 1. - Superfície de resposta (isoquanta = linhas de igual produção, e isóclinas = linhas que cortam as isoquanta em pontos de igual inclinação), indicando para as relaçóes de preços de $N: P=1,5: 1,1,33: 1$ e $1: 1$, as quantidades de nutrientes a serem aplicadas para a obtençáo de malor lucro (ou menor prejuízo) por área, quando forem consideradas três relaçōes de preços entre nutrientes $\left(\mathrm{N}+\mathrm{P}_{2} \mathrm{O}_{5}\right)$ e trigo e mantida constante a dose de $30 \mathrm{~kg} / \mathrm{ha}$ de $\mathbf{K}_{2} \mathrm{O}$.

\begin{tabular}{ccc}
\multicolumn{2}{l}{\begin{tabular}{c} 
kg de trigo $=1$ \\
\multicolumn{2}{l}{ Valores relativos }
\end{tabular}} & $\begin{array}{c}\text { Quantidade a se } \\
\text { aplicada em } \mathrm{kg} / \mathrm{ha}\end{array}$ \\
$\mathrm{K}_{2} \mathrm{O}$ & Trigo & de $\mathrm{K}_{2} \mathrm{O}$ \\
4 & 1 & 63 \\
3 & 1 & 85 \\
2 & 1 & 108 \\
1 & 1 & 130 \\
0 & 0 & 153
\end{tabular}


car-se-ão $108 \mathrm{~kg}$ de $\mathrm{K}_{2} \mathrm{O}$ por hectare; quando for necessário $1 \mathrm{~kg}$ de trigo para comprar $1 \mathrm{~kg}$ de $\mathrm{K}_{2} \mathrm{O}$, aplicar-se-ão $130 \mathrm{~kg}$ de $\mathrm{K}_{2} \mathrm{O}$ por hectare.

Para obter a máxima produção possível por área, não computando os preços dos adubos, aplicar-se-ão $153 \mathrm{~kg}$ de $\mathrm{K}_{2} \mathrm{O} / \mathrm{ha}$.

\section{4 - CONCLUSÕES}

Os resultados obtidos neste trabalho permitem tirar as seguintes conclusões:

a) $\mathrm{O}$ efeito linear do nitrogênio, na média dos dois experimentos, correspondeu a um aumento de $108 \mathrm{~kg}$ por hectare na produção para cada $30 \mathrm{~kg}$ de nitrogênio aplicados.

b) O efeito linear do fósforo, na média dos dois experimentos, correspondeu a um aumento de $189 \mathrm{~kg}$ de trigo por hectare para cada $30 \mathrm{~kg}$ de $\mathrm{P}_{2} \mathrm{O}_{5}$ aplicados.

c) O efeito linear do potássio, na média dos dois experimentos, correspondeu a um aumento de $82 \mathrm{~kg}$ de trigo por hectare para cada $15 \mathrm{~kg}$ de $\mathrm{K}_{2} \mathrm{O}$ aplicados.

d) O efeito isolado do enxotre aumentou a produção média de $11 \%$ em relação à testemunha, e na presença da adubação mineral completa aumentou de $5 \%$ a produção.

e) Considerando a relação atual de preços entre $\mathrm{N}$ e $\mathrm{P}_{2} \mathrm{O}_{5}$ como sendo 1,33:1, as quantidades necessárias de $\mathrm{N}$ e $\mathrm{P}_{2} \mathrm{O}_{\sigma}$ por hectare para obter o maior lucro por área (considerando constante a dose de $30 \mathrm{~kg} / \mathrm{ha}$ de $\mathrm{K}_{2} \mathrm{O}$ ) seriam de $68 \mathrm{~kg}$ e $96 \mathrm{~kg}$, respectivamente.

f) Considerando o preço atual do potássio em relação ao preço do trigo, ter-se-ia que a quantidade de $\mathrm{K}_{2} \mathrm{O}$ por hectare necessária para obter o maior lucro por área (considerando constante as doses de $60 \mathrm{~kg} / \mathrm{ha}$ de $\mathrm{N}$ e $60 \mathrm{~kg} / \mathrm{ha}$ de $\mathrm{P}_{2} \mathrm{O}_{\tilde{z}}$ ) seria de $120 \mathrm{~kg}$. 


\section{FERTILIZER EXPERIMENTS WITH WHEAT (TRITICUM AESTIVUM L.). II.}

\section{SUMMARY}

This work presents the results of two $N, P, K, S$ mineral fertilizer tr:als with wheat (Triticum aestivum L.) carried out at Itapeva, SP, in 1969 and 1971 on Dark Red Latosol soil.

The average results observed in these trials show high effects associated to nitrogen ( $30 \mathrm{~kg} / \mathrm{ha} \mathrm{N}$ increased $118 \mathrm{~kg} / \mathrm{ha}$ in the production), to phosphorus $\left(30 \mathrm{~kg} / \mathrm{ha} \mathrm{P}_{2} 0_{5}\right.$ incr. $\left.189 \mathrm{~kg} / \mathrm{ha}\right)$, and to potassium $\left(15 \mathrm{~kg} / \mathrm{ha} \mathrm{K}_{2} 0\right.$ incr. $82 \mathrm{~kg} / \mathrm{ha}$ ).

Sulphur increased the production in $11 \%$ in relation to the treatment without fertilizer and in $5 \%$ in relation to the complete mineral fertilizer.

An economic study of NPK wheat fertilizing was drawn with the data of the experiments.

\section{LITERATURA CITADA}

1. BOX, G. E.; CONNOR, L. R.; COUSINS, N. R.; DAVIES, O. L.; HIMSNORTH, F. R.\& SILLITTO, G. P. The design and analysis of industrial experiments. London, Oliver and Boyd, 1956. p.495-578.

2. CAmargo, A. P. Aptidāo climática para as culturas da soja, girassol e amendoim no Estado de São Paulo. In: Zoneamento da aptidão ecológica para a cultura da soja, girassol e amendoim do Estado de São Paulo. Projeto 1. INSTIÓLEOS/Secret. Agric. S. Paulo, 1971. p.2-28.

3. CAMARGO, C. E. O. Adubação do trigo. I - Experiências com N, P, K e $\mathrm{S}$ em Latossolo Roxo no Vale do Paranapanema. Bragantia 31:315-324, 1972 .

4. Ensaios regionais. In: Encontro sobre Triticultura, Campinas, 1971. 28p.

5. Relatório dos trabalhos desenvolvidos pelo Setor de Trigo da Seção de Arroz e Cereais de Inverno. Campinas, Instituto Agronômico, 1969 e 1970. (Não publicado)

6. FRATTINI, J. A. Evolução da cultura de trigo. In: Encontro sobre Triticultura, Campinas, 1971 . 83p.

7. IGUE, K.; GARGANTINI, H. \& ALCOVER, M. Efeito da calagem e da adubação fosfatada em solo ácido e de baixa fertilidade, na cultura de trigo. Bragantia 29:59-66, 1970.

8. MIRANDA, L. T. Resultados de experimentos de adubação e sugestões para a interpretação baseada na análise química do solo. In: Cultura e adubação do milho. São Paulo, Instituto Brasileiro de Potassa, 1966. p.451-472.

9. SCARSETH, G. D.; COOK, H. L.; KRANTZ, B. A. \& OHLROGGE, A. J. How to fertilize corn effectively in Indiana. Indiana, Purdue University, 1944. 40p.

10. YAMAGUISHI, C. T. Aspectos econômicos de cultura de trigo no Estado de São Paulo. In: Encontro sobre Triticultura, Campinas, 1971. $12 p$. 